Laser Chem. 1983, Vol. 2, pp. 167-184

0278-6273/83/0206-0167\$12.00/0

(C) harwood academic publishers gmbh

Printed in Great Britain

\title{
Laser Studies of Metal Cluster Beams
}

\author{
R. E. SMALLEY
}

Department of Chemistry and Rice Quantum Institute, Rice University, Houston, Texas 77251

Recent developments in laser and molecular beam technology have now made it possible to produce supersonic beams of virtually any element in the periodic table. Using laser vaporization of the appropriate target, the beam source conditions may be adjusted to produce either the cold free atoms alone, or clusters of these atoms with each other-or with another element. Since the vaporization laser heats only a small spot on the target, extremely high local temperatures can be obtained without heating any other part of the apparatus; and beams of even the highest boiling element (tungsten) are readily obtained both in atomic and cluster form. The . .ysics and chemistry of these exotic cluster species is almost completely unknown on the fundamental level. Even for clusters containing 100 atoms, most of these atoms lie on the surface, and the chemical and physical properties will be predominately surface phenomena. Initial studies of these clusters have entailed the use of one- and twophoton laser ionization with time-of-flight mass selective detection. Using a variety of fixed frequency lasers, the work function of copper clusters has been examined as a function of cluster size in the range from 2 to 29 atoms per cluster. Considerable detailed information has also been obtained for the electronic structure and bond lengths of a number of transition metal dimers and trimers (including $\mathrm{Cu}_{2}, \mathrm{Cr}_{2}, \mathrm{~V}_{2}$, $\mathrm{Mo}_{2}$, and $\mathrm{Cu}_{3}$ ) through the use of high resolution laser spectroscopy with mass-selective photoionization detection.

\section{INTRODUCTION}

The production and detailed study of small metal clusters has become a topic of considerable fascination in the chemical community over the past decade. ${ }^{1-13}$ Aside from the interest that is always expressed in any novel class of molecules, research into the nature of these metal clusters is particularly intense because of their relevance to surface science in general and heterogeneous catalysis in particular. The Fischer-Tropsch catalyst, for example, is composed of microclusters of transition metals such as Fe deposited on a high-surface 
area support such as porous silica or any of a number of synthetic zeolites. Interest in Fischer-Tropsch catalysis has been particularly high in the last decade since it is an example of " $\mathrm{C}_{1}$ chemistry" where the $\mathrm{CO}$ and $\mathrm{H}_{2}$ resulting from gasification of coal is converted to a wide range of hydrocarbons which are otherwise projected to be in short supply as world petroleum resources dwindle.

In spite of this high level of interest, research into the detailed chemistry and physics of small metal clusters is still in its infancy. For most metal cluster species having less than full coordinative saturation with surrounding ligands, the current level of ignorance is almost complete: there is hardly a single fundamental question concerning the structure or dynamics for which the answer is known with certainty. In contrast to other known molecular systems of comparable size, the detailed spectral investigation of metal cluster molecules has been particularly difficult to their extremely complex electronic structure, and often fluxional vibrational behavior. For example, the simplest bare cluster of iron, $\mathrm{Fe}_{2}$, is expected to have over 100 excited electronic states within the first $1 \mathrm{eV}$ above the ground state. ${ }^{14}$ The coordinatively unsaturated clusters (including the bare metal clusters themselves) are highly reactive and are easily perturbed by even the most inert support. Matrix isolation techniques therefore often obscure in this case the very information they are designed to measure.

In view of these facts it is clear that a major advance in the study of metal clusters would be possible if a way was found to generate supersonic beams of the bare clusters cooled to cryogenic temperatures and protected from perturbation by the absence of collisions in the well-collimated beam. As will be discussed below, such a way has now been found.

These bare metal clusters and the adducts they form with various molecular reactants are special and fascinating states of matter. For clusters of size ranging up to over 100 atoms, the majority of the atoms actually will lie on the surface. In the case of most metals, the cohesiveness of this cluster will be sufficiently high that most physical and chemical interactions with the environment will be dominated by the nature of this surface. For this reason the study of metal clusters might best be described as "molecular surface science" since it is the study of the surface-dominated properties of species that are small enough to be treated as discrete molecules, but also large and cohesive enough to have true surfaces. 
Although metals are, by definition, materials with electronic motion that extends over very long, macroscopic distances, it is generally believed that most of the interesting details of surface chemistry and catalysis are a consequence of rather short-range interactions. Since it is currently impossible to treat a macroscopic metal surface in $a b$ initio theory with anywhere near the requisite level of accuracy to predict surface chemistry, many theorists have hoped that the key insights could just as well be modeled by a small cluster of metal atoms near the surface site where the chemistry occurs. ${ }^{9}$ The detailed quantum mechanics of this cluster is calculated accurately and then suitable scaling factors are estimated for the effect of imbedding this cluster in the bulk metal surface. When such calculations are compared with experiment, it is rarely clear what fraction of the error is due to incorrect handling of the cluster (e.g., "was it large enough to represent the active site adequately?", and "was the calculation sufficiently accurate for the assumed cluster size?") as opposed to incorrect handling of the bulk, delocalized phenomena implicit in the imbedding of this cluster back into the macroscopic surface. Molecular beam experiments similar to those described below may soon be able to clarify this issue by providing definitive information as to the true nature of the properties of the free metal cluster as a function of cluster size.

\section{THE LASER VAPORIZATION CLUSTER SOURCE}

The art of generating beams of metal vapors is hardly a new field. Many of the earliest experiments in molecular beams utilized specially designed ovens to generate beams of alkali metals such as sodium and potassium. Since the dimer of these metals exists at appreciable concentration in the equilibrium vapor above the liquid metal at the temperatures used, these oven sources generated quite useful beam intensities for the alkali dimers as well. However, it has been only within the past decade that extensive efforts have been made to extend these simple oven techniques to produce higher clusters of the alkali metals and prepare cluster beams of other, higher boiling metals as well. Schumacher and co-workers were among the first pioneers in this field. ${ }^{15}$ They have been successful in modifying the standard alkali metal beam source such that quite extended cluster 
beams are obtained for sodium and potassium. Using this hot oven supersonic nozzle technology, they have obtained extensive information as to the electronic spectra and ionization thresholds of bare clusters of these alkali metals. Gole and his group have developed this hot oven beam source even further so that somewhat higher boiling metals such as copper can be studied as well. ${ }^{16}$ At the high temperatures required to produce these metal vapor beams, elaborate precautions must be made to ensure that the nozzle housing itself is not destroyed, and that impurities liberated from the hot surfaces do not cause the nozzle orifice to clog. In spite of these difficulties, the Gole group has reported quite usable and stable beam intensities for copper clusters up through the trimer. ${ }^{3}$

More recently, Riley and co-workers at the Argonne National Laboratory have reported quite an impressive oven-based metal cluster beam source that is capable of producing continuous cluster beams of metals such as chromium, nickel, aluminum, copper, and silver, all thermalized to near room temperature. ${ }^{17}$ The technique requires a careful balance of temperature and flow conditions as an inert gas is passed over a hot sample of the desired metal. This low density inert gas + hot metal technique has been a standard approach to metal cluster beam generation for quite a while now-particularly since the early work of Pfund, ${ }^{18}$ Granqvist and Buhrman, ${ }^{19}$ Schultz et al., ${ }^{20}$ Stein and co-workers, ${ }^{21}$ and the groups of Rechnagel ${ }^{22}$ and Andres. $^{23}$

The topic of this paper, however, is an alternative and much more general way to coax metal atoms into the gas phase-a way that avoids ovens entirely, and is equally successful with even the most refractory materials known. The metal is simply vaporized directly off of a room temperature metal target using a pulsed laser. As introduced in a number of short communications and articles over the past few years by the author's group ${ }^{24-28}$ (as well as that of Bondybey ${ }^{29-33}$ ), laser vaporization provides quite a straightforward means of generating metal clusters-at least it is "straightforward" as soon as one has mastered the intricacies of operating pulsed lasers and the requisite number of synchronized triggering and detection devices. Of course, the single greatest advantage is that the laser vaporization process is localized to a small region of the metal target, and nothing else in the apparatus need be heated at all. 
Although we have experimented with a number of competing designs, the laser vaporization cluster source shown schematically in Figure 1 has proved thus far to be the best. Details of this cluster source and its operation have been published a number of times. ${ }^{25,27,28}$ Briefly, this source uses the green second harmonic of a $Q$-switched $\mathrm{Nd}$ :YAG laser (5 ns pulse duration, 10-30 millijoules per pulse) to vaporize the metal. As shown in Figure 1, this vaporization laser beam is focused to a 1-2 mm diameter spot on the surface of a target rod which can either be a solid piece of the desired metal, or an alloy, or electroplated, ... or conceivably even a sintered mixture of a variety of metals or solid metal compounds. In order to prevent the laser from drilling a deep hole in the target, the target rod is slowly rotated and translated using a screw mechanism such that each successive laser shot hits a slightly different area of the surface. The timing of this vaporization laser pulse is such that it coincides with the peak intensity of helium carrier gas flow which is pulsed over the surface of the target rod by a specially-designed fast solenoid-operated valve. As can be seen in the Figure, this flowing carrier gas and the metal plasma generated by the laser are confined within a $2 \mathrm{~mm}$ diameter channel which is bored in the nozzle assembly so that it is

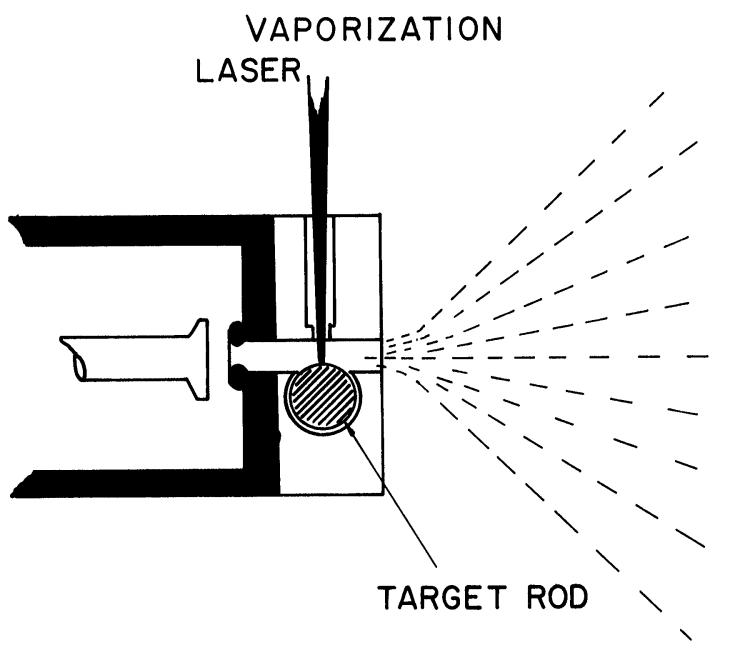

FIGURE 1 Schematic cross-section of the new laser vaporization supersonic metal cluster nozzle. 
tangent to the target rod at the point of laser vaporization. The entire nozzle assembly is operated at room temperature; only the small illuminated region on the target surface is heated by the laser.

Under the conditions typically employed in these experiments (nozzle backing pressure $=4$ atm helium, pulsed nozzle sealing orifice diameter $=1.5 \mathrm{~mm}$ ), the effective carrier gas density over the target at the moment of vaporization is estimated to be on the order of 1-2 atm, and the flow velocity is believed to be only slightly slower than the speed of sound in helium at room temperature (roughly $10^{5} \mathrm{~cm} \mathrm{~s}^{-1}$ ). When the vaporization laser pulse hits the target metal, a metal plasma is produced which becomes so dense during the early part of the laser pulse that it absorbs all further energy from the laser. The light energy in the last part of the laser pulse simply superheats this plasma. In such a strongly irradiated metal plasma, electron temperatures can easily exceed $10000 \mathrm{~K}$, and the metal is entirely in the form of ionized metal atoms. The high density of helium carrier gas surrounding this plasma is sufficient to recombine the electrons with the ions, cool the resultant excited atoms back to near room temperature, and begin the process of condensation through the formation of metal dimers, trimers, and higher clusters. By varying the length of the $2 \mathrm{~mm}$ nozzle channel downstream of the point of laser vaporization, the extent of cluster formation can be controlled within rather broad limits. At the end of this channel, the glas flow becomes supersonic as it freely expands into a large vacuum chamber. Collisions with the helium carrier gas during this supersonic expansion then quite effectively cool the metal clusters to near absolute zero. Collision-free, well-collimated metal cluster beams skimmed from such a pulsed supersonic expansion are then nearly ideal for the detailed spectral study of metal clusters.

Figure 2 displays an example of the cluster distributions typically attained with this type of pulsed laser vaporization metal cluster source for a variety of metals including the extremely high boiling metals molybdenum and tungsten. ${ }^{28}$ These cluster distributions were measured by crossing the molecular beam with the $1930 \mathrm{~A}$ output of an ArF exciplex laser and detecting the resultant photoions using a time-of-flight (TOF) mass spectrometer. The cluster intensity distributions shown in Figure 2 are somewhat misleading since the deflection field used to direct the cluster ions down the TOF mass spectrometer can be optimized only for one mass at a time. In the supersonic 


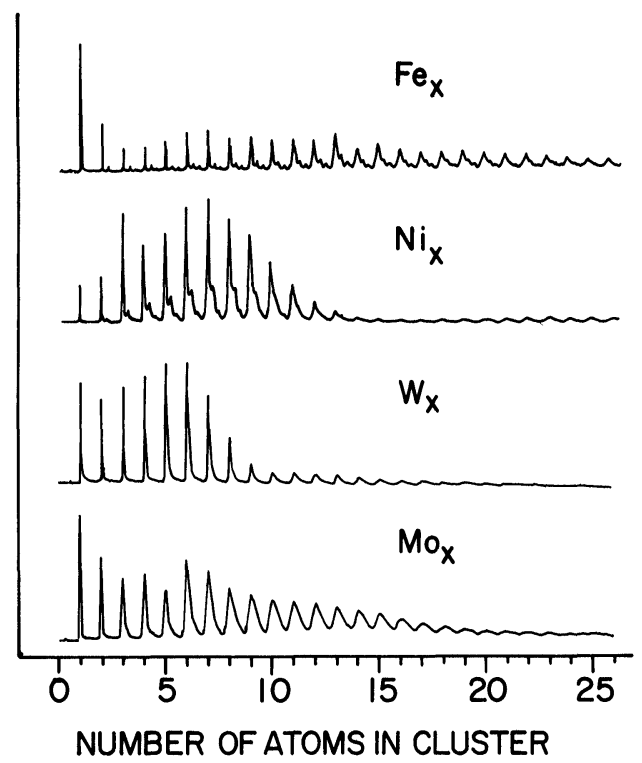

FIGURE 2 Typical metal cluster beam intensity distribution measured for supersonic metal cluster beams produced by the nozzle design shown in Figure 1. An ArF excimer laser was used to photoionize the clusters in the supersonic beam, and the mass spectrum of the resultant photoions is plotted here as a function of the cluster size. The small satellite features seen just to the high mass side of each cluster in the iron and nickel traces are due to oxygen contamination in the laser vaporization source.

expansion all clusters are accelerated up to the helium beam velocity of $1.9 \times 10^{5} \mathrm{~cm} \mathrm{~s}^{-1}$ so that each cluster has a translational energy proportional to its mass. Since the drift tube of the TOF mass spectrometer is perpendicular to the cluster beam, this energy must be removed by a deflection field in order for the ion to make it all the way to the detector. For the mass spectra shown in Figure 2, this deflection field was adjusted to yield optimum detection in the mass range near $500 \mathrm{amu}$. When careful studies are done by successively optimizing for each cluster, it is typically found that the cluster distribution is a monotonically decreasing function of cluster size.

Since the vaporization laser is pulsed over a time period of only $5 \mathrm{~ns}$ and since the nozzle design gives only a smooth, straight flow path for the carrier gas past the metal target, the metal clusters are kept concentrated in a very short time interval of the supersonic 
helium gas pulse. Depending on such details as the operating pressures, the laser pulse energy, and the length of the clustering channel, this time interval in the beam pulse within which the clusters are to be found ranges from 5-20 $\mu \mathrm{s}$. At the helium beam speed this corresponds to only a 1 to $4 \mathrm{~cm}$ length of the pulsed beam that actually contains the clusters. This presents a rather critical demand upon the synchronization of any subsequent laser pulses intended to sample these clusters as they fly through the apparatus. Luckily, modern crystal oscillator-based digital timing electronics is well up to such a task, and the necessary pulse synchronization can be conveniently handled entirely under computer control for all aspects of the experiment.

\section{EVOLUTION OF THE WORK FUNCTION}

One of the most readily measured fundamental properties that can be followed for the metal clusters as a function of cluster size is the ionization potential. As the size of a metal cluster increases, the ionization potential should gradually decrease and asymptotically approach a value which is equal to the work function of the macroscopic metal surface. The over-all rate and functional form of this ionization potential dependence on cluster size is a sensitive test of theoretical predictions of the electronic structure of small clusters.

In the case of copper, the work function is known to lie in the range from $4.6-4.7 \mathrm{eV}$, depending on the plane along which the copper crystal was cleaved. The ionization potential of the copper atom, on the other hand, is known to be $7.72 \mathrm{eV}$. In a resonant two-photon ionization (R2PI) study of a new band system of the copper dimer, the ionization potential of $\mathrm{Cu}_{2}$ was recently determined to be $7.894 \pm 0.015 \mathrm{eV}$, which is slightly higher than the free atom. ${ }^{27}$ Using the new supersonic metal cluster beam source, it will soon be possible to measure the ionization threshold for the intervening clusters in the size range of 3 to roughtly 100 copper atoms by direct one-photon measurement of the photoionization threshold. Due to temporary limitations in tunable UV lasers this has not yet been done, but a crude bracketing of the ionization potentials has been reported using a variety of fixed frequency lasers. ${ }^{27}$ With the $F_{2}$ excimer laser at $1570 \AA$ photoion signal intensity measurements as a function of 
laser intensity have determined that all copper clusters have an ionization potential lower than $7.9 \mathrm{eV}$. The $1930 \AA$ output of an ArF laser, however, produces a copper cluster photoion distribution that provides dramatic evidence of a pronounced alternation in ionization poeential about the $6.4 \mathrm{eV}$ energy of the ArF laser photons. As shown in Figure 3, the even-numbered clusters give rise to far weaker photoion signals than the odd clusters when inoized in a ArF laser beam-particularly for clusters with less than 10 atoms. For the small clusters this is because the even-numbered species have ionization potentials higher than $6.4 \mathrm{eV}$. Signal is seen in the $\mathrm{Cu}_{x}^{+}(x=2,4$, and 6 ) channels only because of a two-photon ionization process, or a small amount of fragmentation from higher mass channels due to the absorption of a second photon by the higher channel parent ion. All odd-numbered copper clusters (with the exception of the monomer) are found to have ionization potentials lower than $6.4 \mathrm{eV}$.

The continued observation of a pronounced even/odd intensity alternation even for the clusters with more than 10 atoms is a secondorder effect. Measurements of the dependence of these signals on laser intensity indicate that all are due to direct one-photon ionization processes, so the ionization potentials of all clusters beyond 10 atoms are less than $6.4 \mathrm{eV}$, regardless of whether the cluster is even or odd.

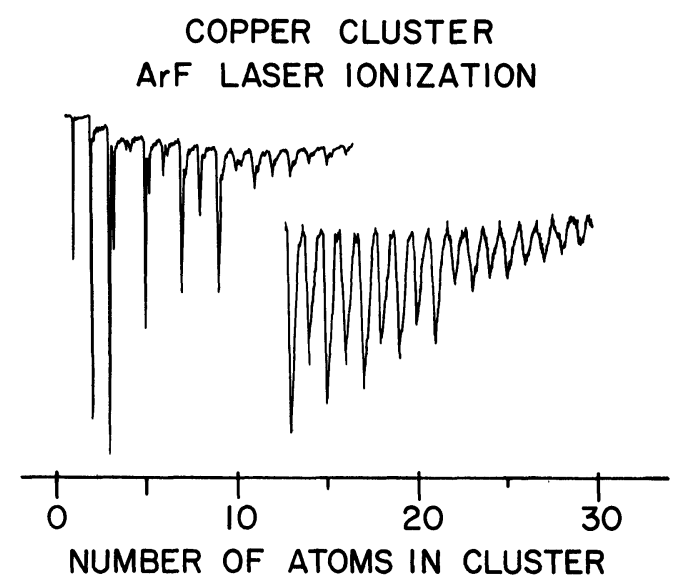

FIGURE 3 Copper cluster photoionization intensity distribution observed using an ArF laser. As in Figure 2, the satellite features are due to oxygen impurities in the laser vaporization source. 
The intensity alternation is believed due to an alternation in the shape of the photoionization threshold region. In even-numbered clusters the highest occupied molecular orbital (HOMO) is doubly occupied and corresponds to a rather strongly bound molecular orbital. Removal of one of these electrons to form the ion produces a rather substantial change in the bonding, and therefore in the geometry and vibrational frequencies between the ion and the parent neutral. Franck-Condon factors then force the photoionization cross-section in the threshold region to be a rather slowly rising function for the ven-numbered clusters. The odd-numbered clusters, on the other hand, have a HOMO that is singly-occupied and approximately nonbonding. Ionization out of this orbital in the odd clusters therefore results in little geometry change, and the photoionization threshold regions is nearly a step function at the true ionization potential.

Such an alternation in the electronic structure of copper clusters is just what is expected if the dominant bonding interaction involves primarily the $4 s$ atomic orbital on each copper atom with the $d$ band of the metal cluster being quite narrow and far deeper than the top of the wide $4 s$ band which is responsible for the observed threshold ionization behavior. Current $a b$ initio calculations reproduce this alternation effect quite well, although the quantitative agreement with the actual ionization potentials is still rather poor. Experiments similar to that sown here for ArF laser radiation at $6.4 \mathrm{eV}$ have been done at a number of lower photon energies. The results indicate the ionization potential has still not fallen below $4.98 \mathrm{eV}$ for $\mathrm{Cu}_{29}$.

These ionization potential measurements are rather crude, and more satisfactory direct and continuous scans will soon be made of the photoionization threshold region for each cluster. However, the current bracketing results are already sufficient to provide critically needed bench marks for further theoretical calculations.

\section{SPECTRAL PROBES OF CLUSTER STRUCTURE}

For roughly a decade now there has been an exponentially increasing level of publication activity attesting to the power of laser spectroscopy in supersonic molecular beams. The extensive cooling to just a few populated quantum states, and the collision-free beam environment are ideal for ferreting out incredibly detailed structural and dynamic 
information on large polyatomic molecules. With this new laser vaporization cluster source it is expected that laser spectroscopy of metal clusters will be equally rewarding.

\section{Copper dimer and trimer}

The first indication that the metal clusters in the pulsed supersonic beam were indeed extremely cold came in a resonant two-photon ionization (R2PI) experiment on the copper dimer. ${ }^{25}$ Here the first photon came from a pulsed dye laser that was scanned over the known $B^{1} \Sigma_{u}^{+} \leftarrow X^{1} \Sigma_{g}^{+}$electronic absorption near $4600 \AA$. The second photon came from an ArF exciplex laser operating at $1930 \AA$. This second photon was of sufficient energy to directly photoionize the electronically excited ( $B$ state) copper dimers, but could only weakly photoionize the ground state molecules by a non-resonant coherent multiphoton route. By keeping the ArF laser intensity low so as to minimize multiphoton ionization, the excited $B$ state population produced by the scanning dye laser could be easily detected. A major advantage of this R2PI scheme for measuring the $B \leftarrow X$ spectrum is that the mass-selective detection not only discriminates effectively against spectral features originating in cluster species other than that of interest, but it also provides automatically a full isotopic resolution of the spectrum.

In the case of the copper dimer, the measured rotational temperature in the supersonic beam was $4 \mathrm{~K}$. Vibrational degrees of freedom are known to be difficult to cool in supersonic expansionsparticularly for small diatomics and triatomics where the vibrational frequencies are fairly high and the vibrational level density is extremely low. Even so, in the case of $\mathrm{Cu}_{2}$ the $v=1$ level population $\left(w_{e}=265 \mathrm{~cm}^{-1}\right)$ was found to correspond to a vibrational temperature between 20 and $70 \mathrm{~K}$, depending on the exact operating parameters of the nozzle. Some population of higher vibrational levels was also noted (with small but still detectable population in levels all the way up to $v=10$ ) and the relative populations did not fit a Boltzman distribution. This failure to reach thermal equilibration in vibrational degrees of freedom is a common result for small molecules in free jet expansions of the intensity attained from this metal cluster nozzle.

Even though diatomic metal clusters are difficult to cool vibrationally, this presents no particular problem since the diatomic spectra 
are usually simple enough that the extra vibrational structure can easily be resolved and used to provide new information. Such a vibrational cooling problem would be far more prohibitive in a larger cluster where the overlapping of vibrational hot bands could easily obscure the entire spectrum. Luckily, it often turns out that the vibrational cooling problem essentially vanishes as soon as the molecule is large and/or floppy enough to have a high density of vibrational states at low total energy - which is just where the absence of effective vibrational cooling would have made for terrible problems in the spectroscopy.

In fact, a fine example of this last point is found in the next higher cluster of copper, $\mathrm{Cu}_{3}$, which has recently been studied in this same supersonic cluster beam apparatus. ${ }^{34}$ For this somewhat more complicated cluster the vibrational cooling is sufficient that only one "hot band" has been observed and its intensity is only $5 \%$ of the parent cold band. The observed spectrum of $\mathrm{Cu}_{3}$ has now been conclusively assigned as the $\tilde{A}^{2} E^{\prime} \leftarrow \tilde{X}^{2} E^{\prime}$ transition of a $D_{3 h}$ triatomic exhibiting strong Jahn Teller distortion in both the ground and excited electronic state. In the ground state only the lowest few vibrational levels are below the saddle point (corresponding to an obtuse isosceles distortion) for introconversion among the three equivalent acute isosceles minima on the Jahn-Teller distorted potential surface. The $\mathrm{Cu}_{3}$ cluster is therefore highly fluxional even at room temperature, and possesses a very complicated vibrational level structure. Without the extensive vibrational cooling provided by the supersonic expansion, resolution and assignment of the electronic spectrum may very well have been a hopeless task.

\section{Chromium and molybdenum dimers}

One of the more intense areas of intellectual ferment in the current quantum chemical community lies in the understanding of the metalmetal bond. Even for the simplest case-a bare homonuclear diatomic molecule-the requirement that $d$ orbitals be considered, coupled with the need to consider many open shell configurations of high spin and, for heavier atoms, the necessity of making relativistic corrections renders the metal-metal bonding problem very challenging indeed. The solution of this problem is, of course, only one of the first steps on the road to reliable ab initio predictions of surface chemistry. 
In this issue of metal-metal bonding the dimers of the Group VI transition metals (Cr, Mo, and $\mathrm{W})$ are particularly interesting. For both $\mathrm{Cr}$ and Mo the ground state electronic configuration is a ${ }^{7} S$ state which arises from one unpaired electron in each of the five $d$ orbitals and one in the valence $s$ orbital. When two such atoms come together at sufficiently close distances, it is conceivable that as many as six bonds could form to produce a tightly bound ${ }^{1} \Sigma_{\mathrm{g}}^{+}$state (of electronic configuration $\sigma_{3 d}^{2} \pi_{3 d}^{4} \delta_{3 d}^{4} \sigma_{4 s}^{2}$ ). In fact, such a configuration has been calculated for the $\mathrm{Mo}_{2}$ dimer in a recent extensive generalized valence bond (GVB) treatment by Goodgame and Goddard. ${ }^{35}$ However, this same theoretical technique predicts a very weak bond with a $3.0 \AA$ bond length for $\mathrm{Cr}_{2}$. Here the GVB theory predicts the bonding to be almost entirely due to a weak $4 s$ orbital sigma bond, with little $d$ orbital participation. ${ }^{36}$ Recent calculations by Atha and Hillier have confirmed this prediction. ${ }^{37}$ Far from having a sextuple bond, the dimer of chromium is predicted to be better understood as an antiferromagnetically coupled van der Waals dimer of two ${ }^{7} S$ atoms.

Using the new supersonic metal cluster beam source, this issue has now been resolved. ${ }^{26}$ Figure 4 shows a rotationally resolved spectrum of a strong vibronic band found in the $4600 \AA$ region for $\mathrm{Cr}_{2}$ in the supersonic beam. Since this spectrum was taken by the mass-selective

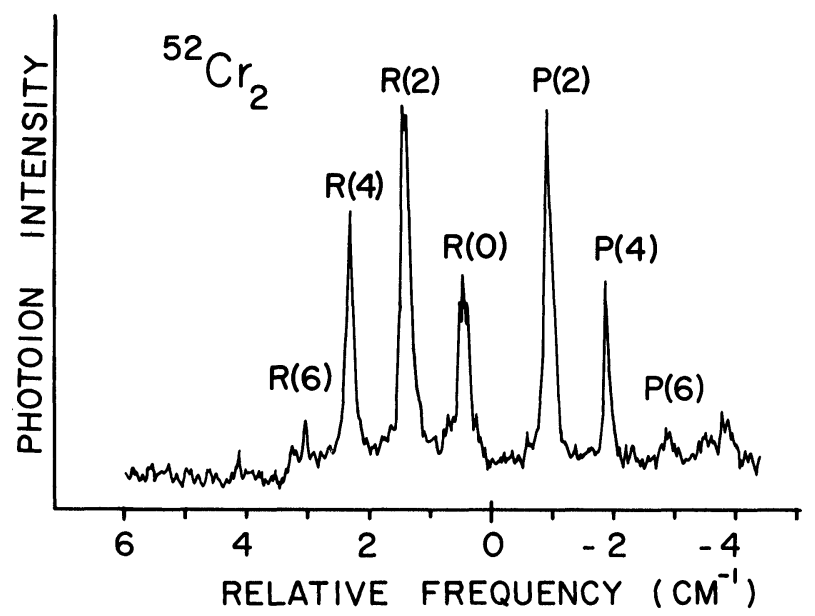

FIGURE 4 Rotationally resolved spectrum of the 0-0 band of the $A^{1} \Sigma_{u}^{+} \rightarrow X^{1} \Sigma_{g}^{+}$ electronic transition of the chromium dimer cooled in a supersonic beam. The zero of the relative frequency scale corresponds to $21751 \mathrm{~cm}^{-1}$. 
R2PI technique, it was quite straightforward to record simultaneously separate spectra for the various isotopic variants of this dimer. The spectrum shown in the Figure applies to the 52-52 isotope, and the absence of a $Q$ branch in the spectrum together with the fact that levels in the lower electronic state with odd values of the angular momentum, $J$, are missing, indicates that the spectrum is due to the $A^{1} \Sigma_{u}^{+} \leftarrow X^{1} \Sigma_{g}^{+}$electronic transition of a homonuclear diatomic with zero nuclear spin. Measurement of the line positions shows that the internuclear bond distance in the ground ${ }^{1} \Sigma_{g}^{+}$state is $1.68 \AA$. Far from the weakly-bound antiferromagnetic dimer predicted by theory, this turns out to be the shortest formal bond distance of any known homonuclear diatomic (for comparison, the nearest neighbor $\mathrm{Cr}-\mathrm{Cr}$ distance in metallic chromium is $2.5 \AA$. At such a short internuclear separation there is very extensive $d$ orbital overlap, and there can be little doubt that the bonding in $\mathrm{Cr}_{2}$ is, in fact, dominated by $d$ orbital bonding interactions.

Much the same story applies to the molybdenum dimer. ${ }^{28}$ Here too a very strong, sharp vibronic band is found in the visible (5180 $\AA$ ), and its rotational structure reveals it to arise from an $A^{1} \Sigma_{u}^{+} \leftarrow X^{1} \Sigma_{\mathrm{g}}^{+}$ transition where the gound state has a very short bond length $(1.94 \AA)$ which can only be explained by very strong bonding involving the $d$ orbitals. As in $\mathrm{Cr}_{2}$, the upper electronic state in this transition of the molybdenum dimer has virtually the identical bond length as the ground state (to within a few parts in a thousand). This is highly unusual among homonuclear diatomics and strongly supports the conclusion that the electronic structure of $\mathrm{Cr}_{2}$ and $\mathrm{Mo}_{2}$ are identical.

There is an interesting side light to this story in that quite strongly suggestive experimental evidence existed for these conclusions far before this recent high-technology beam study. Efremov and coworkers observed the same vibronic bands as discussed above for both the chromium and molybdenum dimers, and reported the results of a rotational analysis. ${ }^{38-39}$ In both cases the spectrum was generated by flash-photolyzing the hexacarbonyl vapor of the transition metal, and recording the transient absorption of the room-temperature dimer with a conventional spectrograph. Since there was no isotopic analysis, and no concurrent mass analysis to firmly label the carrier of the spectra, they could only offer plausibility arguments for their assignment. The matrix isolation work of Ozin actually gave striking support to the assignment, ${ }^{40-41}$ and in retrospect it is rather hard to see how 
any other assignment for the carrier of the spectrum could have adequately explained both the gas phase and matrix results. Nonetheless, because the spectra indicated the $\mathrm{Cr}_{2}$ bond was so incredibly short and because such a short bond did not fit in well with the theories currently in vogue, these conventional spectral results were widely disregarded. In the end it took overwhelming technology to settle the issue.

\section{Vanadium dimer}

As a final example of the use of laser spectroscopy with the new supersonic cluster source in the study of metal cluster structure and bonding, consider a somewhat more complicated dimer: $V_{2}$. Figure 5 displays a low resolution spectrum of an assembly of vibronic bands for $V_{2}$ in the $6700-7600 \AA$ region as measured by the mass-selective R2PI technique. Most of the major bands seen here have now been rotationally analyzed. They all turn out to involve an ${ }^{3} \Pi_{u} \leftarrow{ }^{3} \Sigma_{g}^{-}$ electronic transition where ${ }^{3} \Sigma_{g}^{-}$appears to be the ground state. It would be the state symmetry expected from an electronic configuration which left two unpaired electrons in the degenerate $\delta_{3 d}$ orbital, i.e., $\left(\sigma_{3 d}^{2} \pi_{3 d}^{4} \sigma_{4 s}^{2} \delta_{3 d}^{2}\right)$. The singlet state $\left({ }^{1} \Sigma\right)$ arising from the same configuration must be rather close by, since the spectrum shown in Figure 5 can only be explained if the ${ }^{3} \Sigma_{g}^{-}$state is split into its $\Omega=0$

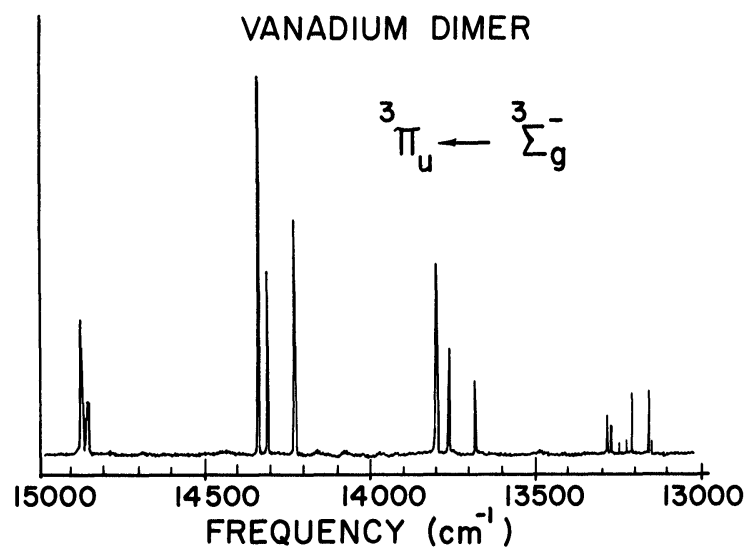

FIGURE 5 Low resolution spectrum of the vanadium dimer in the supersonic beam, showing several vibrational bands of the ${ }^{3} \pi_{u} \leftarrow{ }^{3} \Sigma_{g}^{-}$electronic transition. 
and $\Omega=1$ components by $75 \mathrm{~cm}^{-1}$ for $v=0$ and $85 \mathrm{~cm}^{-1}$ for $v=1$. Similar strong spin-orbit interactions are known in other cases where there is a half-filled $\pi$ or $\delta$ orbital such as in $\mathrm{S}_{2}$ and $\mathrm{Se}_{2}$. As in the case of $\mathrm{Cr}_{2}$, vanadium's dimer is found to have an extremely short bond length (1.76 $\AA$ ), although here the electronic excitation to the upper state actually produces a readily measurable bond shortening (to $1.69 \AA$ ). The major vibrational intervals seen in Figure 5 are due to a pronounced hot band progression which shows the vibrational frequency in the ground state to be $539 \mathrm{~cm}^{-1}$ which constitutes the current all-time record for the vibrational frequency of a diatomic metal. As in chromium and molybdenum, this extremely short bond length and high vibrational frequency is strong evidence for the dominance of $d$ electrons in the bonding of these transition metals.

\section{CONCLUSION}

The recently developed laser vaporization beam source has provided an exceptionally fine opportunity to obtain detailed insight into the physics and chemistry of small metal clusters. It is now obvious that one major area of impact will be in the understanding of the metalmetal bond. But, in the long run, this early concentration on molecular spectroscopy of dimers and trimers is likely to be seen as an important, but relatively esoteric side activity. The issues raised in the introduction related to the surface physics and chemistry of these exceptional state of matter will require a substantially different approach than the molecular spectroscopy used thus far. Certainly the excitement and challenge in this field is just beginning.

\section{Acknowledgement}

The work featured in this article was performed by a set of superb graduate students and postdoctoral associates whose names are cited in the appropriate citations for each set of experiments. Professor A. J. Merer was a critical factor in the assignment and understanding of the vanadium dimer spectrum mentioned briefly above. The research at Rice in the area of metal clusters is funded by the Division of Chemical Sciences of the Office of Basic Energy Sciences of the US Department of Energy, and by the Robert A. Welch Foundation. 


\section{References}

1. E. L. Muetterties, Chem. Eng. News. 60 (35), 28 (1982).

2. F. A. Cotton and M. H. Chisholm, Chem. Eng. News. 60 (26), 40 (1982).

3. J. L. Gole and W. C. Stwalley, eds., Metal Bonding and Interactions in High Temperature Systems, ACS Symposium Series 179 (Am. Chem. Soc., Washington, D.C., 1982).

4. G. L. Geoffroy, Accts. Chem. Res. 13, 469 (1980).

5. E. L. Muetterties, T. N. Rhodin, E. Bond, C. F. Bruckner and W. R. Pretzer, Chem. Rev. 79, 91 (1979).

6. G. A. Ozin, M. P. Andrews, L. F. Nazar, H. X. Huber and C. G. Francis, Coordination Chem. Rev. in press.

7. G. A. Ozin, and S. A. Mitchell, Angew. Chemie Int. Ed. in press.

8. W. C. Trogeler and H. B. Gray, Acc. Chem. Res. 11, 223 (1978).

9. R. P. Messmer, in: The Nature of the Surface Hemical Bond eds. T. N. Rhodin and G. Ertl (North Holland, Amsterdam, 1979) Chapter 2.

10. Proceedings of the Second International Meeting on Small Particles and Inorganic Clusters, Surf. Sci. 106 (1981).

11. J. H. Sinfelt, Acc. Chem. Res. 10, 15 (1977).

12. M. Moskovits, Acc. Chem. Res. 7, 229 (1979).

13. H. F. Schaefer, III, Acc. Chem. Res. 10, 287 (1977).

14. I. Shim and K. A. Gingerich, J. Chem. Phys. 77, 2490 (1982).

15. E. Schumacher, W. H. Gerber, H.-P. Harri, M. Hofmann and E. Scholl, ACS Symposium Series 179, 83 (1982).

16. D. P. Preuss, S. A. Pace and J. L. Gole, J. Chem. Phys. 71, 3553 (1979).

17. S. J. Riley, E. K. Parks, C. R. Mao, L. G. Pobo and S. Wexler, J. Phys. Chem. 86, 3911 (1982).

18. A. H. Phund, Phys. Rev. 35, 1434 (1930); Rev. Sci. Instrum. 1, 397 (1930).

19. C. G. Granqvist and R. A. Buhrman, J. Appl: Phys. 47, 2200 (1976).

20. W. Schultz, H. O. Becker, R. Minkwitz and K. Manzel, Chem. Phys. Lett. 55, 59 (1978).

21. (a) A. Yokozeki and G. D. Stein J. App. Phys. 49, 2224 (1978); (b) B. G. DeBoer and G. D. Stein Surface Science 106, 84 (1981); (c) A. Yokozeki and G. D. Stein J. Chem. Phys. 49, 2224 (1978).

(a) J. Muhlbach. E. Rechnagel and K. Sattler, Surface Science 106, 188 (1981); (b) O. Echt, K. Sattler and E. Rechnagel, Phys. Rev. Lett. 47, 1121 (1981).

23. R. S. Bowles, J. J. Kolstad, J. M. Calo and R. P. Andres, Surface Science 106, 117 (1981).

24. T. G. Dietz, M. A. Duncan, D. E. Powers and R. E. Smalley, J. Chem. Phys. 74 $6511(1981)$.

25. D. E. Powers, S. G. Hansen, M. E. Geusic, A. C. Puiu, J. B. Hopkins, T. G. Dietz, M. A. Duncan, P. R. R. Langridge-Smith and R. E. Smalley, J. Phys. Chem. 862556 (1982).

26. D. L. Michalopoulos, B. E. Geusic, S. G. Hansen, D. E. Powers and R. E. Smalley, J. Phys. Chem. 863914 (1982).

27. D. E. Powers, S. G. Hansen, M. E. Geusic, D. L. Michalopoulos and R. E. Smalley, J. Chem. Phys. 782866 (1983).

28. J. B. Hopkins, P. R. R. Langridge-Smith, M. D. Morse and R. E. Smelley, J. Chem. Phys. 78, 1627 (1983).

29. V. E. Bondybey and J. H. English, J. Chem. Phys. 74, 6978 (1981).

30. V. E. Bondybey and J. H. English, J. Chem. Phys. 76, 2165 (1982).

31. V. E. Bondybey, J. Chem. Phys. 77, 3771 (1982). 
32. V. E. Bondybey, J. Phys. Chem. 86, 3396 (1982).

33. M. Heaven, T. A. Miller, J. H. English and V. E. Bondybey, Chem. Phys. Lett. 91, 251 (1982).

34. M. D. Morse, J. B. Hopkins, P. R. R. Langridge-Smith and R. E. Smalley, J. Chem. Phys. submitted.

35. M. M. Goodgame and W. A. Goddard, Phys. Rev. Lett. 48, 135 (1982).

36. M. M. Goodgame and W. A. Goddard, J. Phys. Chem. 85, 215 (1981).

37. P. M. Atha and I. H. Hillier, Mol. Phys. 45, 285 (1982).

38. Yu. M. Efremov, A. N. Samoilova, V. B. Kozhukhovsky and L. L. V. Gurvich, J. Mol. Spectrosc. 73, 430 (1978).

39. Yu. M. Efremov, A. N. Samoilova and L. V. Gurvich, Opt. Spectrosc. 36, 381 (1974).

40. G. A. Ozin, W. Klotzbucher, J. G. Norman, Jr. and H. J. Kolari, Inorg. Chem. 16, 2871 (1977).

41. G. A. Ozin and W. Klotzbucher, Inorg. Chem. 16, 984 (1977). 